Artículo de posesión

\title{
Una convolución muy útil y unas derivadas ilustres
}

\author{
(1) Carlos E. Mejía \\ Escuela de Matemáticas, Universidad Nacional de Colombia, Medellín, Colombia \\ Artículo de posesión para el ingreso como miembro correspondiente a la \\ Academia Colombiana de Ciencias Exactas, Físicas y Naturales el 23 de julio del 2019
}

\begin{abstract}
Resumen
En el artículo se exponen aspectos importantes sobre los operadores de molificación discreta y las derivadas fraccionarias. Los operadores de molificación se definen a partir de convoluciones con núcleos gaussianos truncados en una o en dos dimensiones. Se incluye aquí una descripción de sus orígenes y de sus principales propiedades, y se describen en detalle dos aplicaciones que indican su gran utilidad. Las aplicaciones se basan en ecuaciones diferenciales parciales difusivas con derivadas temporales fraccionarias, derivadas que, como se explicará más adelante, merecen el calificativo de ilustres. La primera aplicación consiste en la solución estable de un problema inverso de advección-dispersión con derivada temporal fraccionaria, en el que la concentración es desconocida en la frontera de un dominio unidimensional semiinfinito. La segunda aplicación es la solución estable de un problema inverso bidimensional de identificación de un término fuente en una ecuación de difusión con derivada temporal fraccionaria. En cada caso se incluye la descripción del problema, la implementación de la molificación, el método de solución y algunos experimentos numéricos. En cuanto al problema en dos dimensiones, se incluyen resultados recientemente publicados.
\end{abstract}

Palabras clave: Convolución; Derivadas fraccionarias; Molificación discreta; Problemas inversos.

\section{A very useful convolution and some illustrious derivatives}

\begin{abstract}
This paper deals with discrete mollification operators and fractional derivatives. The mollification operators are based on convolutions with truncated Gaussian kernels in one and two dimensions. We begin with a description of their origin and main properties and then we consider two applications that show the usefulness of these operators. Both applications are based on time-fractional diffusion equations. Fractional derivatives deserve to be called illustrious, as we will see later. The first application consists of the stable solution of an inverse problem for a time fractional advection-dispersion equation. The problem consists of the identification of the boundary concentration in a one-dimensional semi-infinite setting. The second application is the stable solution of a problem of source term identification in a bidimensional time-fractional diffusion equation. In each case, we include a description of the problem, the mollification implementation, the method of solution, and some numerical experiments. For the two dimensions problem we include results that were recently published.
\end{abstract}

Key words: Convolution; Fractional derivatives; Discrete mollification; Inverse problems.

\section{Introducción}

La palabra "molificar" significa "suavizar" y fue introducida en la matemática en un artículo de Friedrichs (1944). Así, hacer la convolución de una función con un núcleo gaussiano se denomina molificación y ciertamente tiene un efecto suavizante o regularizador. En este artículo nos referimos a una forma específica de suavizar funciones que consiste en calcular su convolución discreta con pesos definidos a partir de una campana gaussiana truncada. Dicha convolución, denominada "molificación discreta", se puede definir en una y en dos dimensiones. El poder regularizador de la convolución con un núcleo gaussiano fue descrito por primera vez por Manselli \& Miller (1980). Miller explicó su idea de molificación a su estudiante doctoral Diego A. Murio y fue este quien emprendió la tarea de utilizar la molificación en una amplia serie de problemas, pues Manselli y Miller declinaron continuar con el tema y se dedicaron a otros asuntos.

La utilidad de la molificación discreta se ha comprobado en una gran variedad de problemas unidimensionales, como los descritos por Mejía (2007), Acosta \& Mejía (2014),

\footnotetext{
Correspondencia:

Carlos E. Mejía; cemejia@unal.edu.co

Recibido: 29 de septiembre de 2018

Aceptado: 7 de junio de 2019

Editor: Jaime Lesmes Camacho
} 
Murio (1993, 2002.) Las aplicaciones que se presentan a continuación son de desarrollo reciente y corresponden a un tema de investigación muy actual, pues la generalización del operador de molificación en dos dimensiones y la inclusión de derivadas fraccionarias en las ecuaciones abrieron un importante campo de acción a quienes trabajamos con la molificación.

El presente artículo se organizó de la siguiente manera: la primera sección se ocupa de las definiciones y las propiedades de la molificación discreta y las ilustres derivadas fraccionarias. En la siguiente sección se considera un problema inverso unidimensional de recuperación de información en la frontera de un dominio semiinfinito para una ecuación difusiva con derivada temporal fraccionaria. Se continúa con una sección centrada en un problema inverso bidimensional consistente en la identificación de un ingrediente dentro de un término fuente, también para una ecuación difusiva con derivada temporal fraccionaria, en tanto que la última sección está dedicada a la discusión y las conclusiones.

\section{Principales operadores}

En la primera parte de esta sección se define el operador de molificación discreta en una dimensión y su generalización a dos dimensiones. En la segunda parte, se introducen las derivadas fraccionarias, se explica el por qué se las llama ilustres y se consideran brevemente algunas de sus aplicaciones.

Molificación discreta unidimensional. El método de molificación es un procedimiento de filtrado basado en la convolución con un núcleo gaussiano truncado que sirve para regularizar problemas mal condicionados y para estabilizar y evitar oscilaciones ficticias en la discretización de ecuaciones diferenciales parciales por medio de esquemas explícitos.

Sea $y=\left\{y_{j}\right\}_{j \in \mathbb{Z}}$ una función discreta, por ejemplo, evaluaciones o promedios de una función real $y=y(x)$, definida en una malla de puntos equidistantes

$$
X=\left\{x_{j}: x_{j}=x_{0}+j h, j \in \mathbb{Z}\right\},
$$

donde $x_{0}$ y $h$ son reales y $h$ es positivo. La molificación de esta función discreta es la convolución

$$
\left(J_{y}\right)_{j}:=\sum_{i=-\eta}^{\eta} \omega_{i} y_{j-i}
$$

donde $\eta$ es el parámetro de soporte y los factores de ponderación o pesos satisfacen

$$
\omega_{i}=\omega_{-i}, 0 \leq \omega_{i} \leq \omega_{i-1}, i=1, \ldots, \eta ; \sum_{i=-\eta}^{\eta} \omega_{i}=1, \quad \sum_{i=-\eta}^{\eta} i \omega_{i}=0 .
$$

Para calcular los pesos se utiliza un núcleo gaussiano truncado que se construye con base en dos números reales positivos, $p$ y $\delta$ que satisfacen la igualdad $\delta=\frac{\eta+\frac{1}{2}}{p}$. El núcleo es

$$
\kappa_{p \delta}=\left\{\begin{array}{cc}
A_{p} \delta^{-1} \exp \left(-\frac{t^{2}}{\delta^{2}}\right), & |t| \leq p \delta \\
0, & |t|>p \delta,
\end{array}\right.
$$

donde la constante de normalización $A_{p}=\left(\int_{-p}^{p} \exp \left(-s^{2}\right) d s\right)^{-1}$ es tal que $\int_{\mathbb{R}} \kappa_{p \delta} d x=1$. El núcleo tiene soporte compacto, pues es cero fuera del intervalo $[-p \delta, p \delta]$. En adelante suponemos que $p=3$. El número $p$ determina el soporte del núcleo y el número $\delta$ se encarga de la forma de la campana gaussiana, mientras mayor es $\delta$ más aplanada es la campana. Los pesos se definen asi: $\omega_{i}=\int_{t_{i}}^{t_{i+1}} \kappa_{p \delta}(-s) d s$, donde $t_{i}=\left(i-\frac{1}{2}\right) h, i \in \mathbb{Z}$. Nótese que estos pesos son independientes de $h$ pues $\frac{t_{i}}{\delta}=p \frac{\left(i-\frac{1}{2}\right) h}{\left(\eta+\frac{1}{2}\right) h}=p \frac{\left(i-\frac{1}{2}\right)}{\left(\eta+\frac{1}{2}\right)}$ es decir, los pesos no dependen del parámetro de discretización espacial, ya que

$$
\begin{gathered}
\omega_{i}=\int_{t_{i}}^{t_{i+1}} \kappa_{p \delta}(-s) d s=A_{p} \delta^{-1} \int_{t_{i}}^{t_{i+1}} \\
\exp \left(-\frac{s^{2}}{\delta^{2}}\right) d s=A_{p} \int_{\frac{t_{i}}{\delta}}^{\frac{t_{i+1}}{\delta}} \exp \left(-s^{2}\right) d s .
\end{gathered}
$$

La tabla 1 muestra una lista de pesos.

La Figura 1 ilustra la suavización que genera la molificación cuando se aplica a una función ruidosa. Más precisamente, sea

$$
f(x)=\left\{\begin{array}{cc}
2 x, & 0 \leq x \leq \frac{1}{2} \\
-2(x-1), & \frac{1}{2} \leq x \leq 1 \\
0, & x \notin[0,1] .
\end{array}\right\}
$$

La función $f$ y su molificación, que denotaremos $J f$ aparecen en la primera gráfica de la figura 1. Por otro lado, sea $\varepsilon>0$ y sea $f^{\varepsilon}$ una aproximación de $f$ que cumple $\| f$ $f^{\varepsilon} \|_{\infty} \leq \varepsilon$. La función aproximada se denomina "ruidosa", pues se obtiene sumando a cada punto un número aleatorio distribuido uniformemente en el intervalo $[-\varepsilon, \varepsilon]$. A una tal distribución de números aleatorios se le conoce como "ruido" gaussiano.

\begin{tabular}{|c|c|c|c|c|c|c|}
\hline$\eta$ & $\omega_{0}$ & $\omega_{1}$ & $\omega_{2}$ & $\omega_{3}$ & $\omega_{4}$ & $\omega_{5}$ \\
\hline 1 & $8.4272 \mathrm{e}-1$ & $7.8640 \mathrm{e}-2$ & & & & \\
\hline 2 & $6.0387 \mathrm{e}-1$ & $1.9262 \mathrm{e}-1$ & $5.4438 \mathrm{e}-3$ & & & \\
\hline 3 & $4.5556 \mathrm{e}-1$ & $2.3772 \mathrm{e}-1$ & $3.3291 \mathrm{e}-2$ & $1.2099 \mathrm{e}-3$ & & \\
\hline 4 & $3.6266 \mathrm{e}-1$ & $2.4003 \mathrm{e}-1$ & $6.9440 \mathrm{e}-2$ & $8.7275 \mathrm{e}-3$ & $4.7268 \mathrm{e}-4$ & \\
\hline 5 & $3.0028 \mathrm{e}-1$ & $2.2625 \mathrm{e}-1$ & $9.6723 \mathrm{e}-2$ & $2.3430 \mathrm{e}-2$ & $3.2095 \mathrm{e}-3$ & $2.4798 \mathrm{e}-4$ \\
\hline
\end{tabular}

En la segunda gráfica de la Figura 1 aparecen $f^{\varepsilon}$ y $J f^{\varepsilon}$. El código MATLAB utilizado para generar las dos funciones $f$ y $f^{\varepsilon}$ para $\varepsilon=0.1$, es el siguiente:

$\mathrm{f}=@(\mathrm{x}) 2 * \mathrm{x} . *(\mathrm{x}<0.5 \& 0<\mathrm{x})+(2-2 * \mathrm{x}) . *(0.5<\mathrm{x} \& \mathrm{x}<1)$; $\mathrm{x}=$ linspace $(-.2,1.2)$;

Tabla 1. Pesos de molificación 
$f x=f(x)$;

epsil $=0.1$;

fep $=f x+(2 * \operatorname{rand}(\operatorname{size}(x))-1) *$ epsil;

Algunos ejemplos de problemas mal condicionados en los que se puede trabajar con molificación son los inversos de difusión (Mejia \& Murio, 1996; Murio, 2007; Murio \& Mejía, 2008a; Mejía \& Piedrahita, 2017) y los inversos de identificación de modelos (Mejia \& Murio, 1995; Murio \& Mejía, 2008b; Mejía, et al., 2011; Acosta, et al., 2014; Acosta, et al., 2015; Echeverry \& Mejía, 2018).

La mayoría de las veces trabajamos con funciones definidas en intervalos, lo cual hace que la definición de molificación, por ser una convolución, no se pueda usar en algunos puntos vecinos de los extremos del intervalo. Esta dificultad se discute en el trabajo de Acosta \& Mejía (2008), en el que se definen las llamadas condiciones de borde, las cuales consisten en modificaciones del operador de molificación para esos puntos limítrofes. A pesar del éxito de tales modificaciones, este es un aspecto de la molificación que aún requiere más estudio.

A manera de conclusión podemos decir que la molificación es una buena alternativa como método de regularización. Además, es una herramienta para hacer menos estrictas las condiciones de estabilidad del tipo CourantFriedrichs-Lewy (CFL), que esperamos que siga vigente por un buen tiempo. Este uso de la molificación se puede consultar en Acosta, et al. (2012, 2014, 2015).

Las propiedades de aproximación de la molificación discreta se pueden consultar en Acosta \& Mejía (2014). Hay aspectos de la molificación que requieren profundización, por ejemplo, las condiciones de borde y la escogencia de los parámetros $\delta$ o $\eta$, que sería deseable hacer de forma automática según cuán perturbados estén los datos que se van a molificar.

Existen otras versiones del método de molificación, por ejemplo, las de Garshasbi \& Dastour (2015), Hao (1994), Li \& Fu (2011) y Shi, et al. (2016), las cuales se diferencian

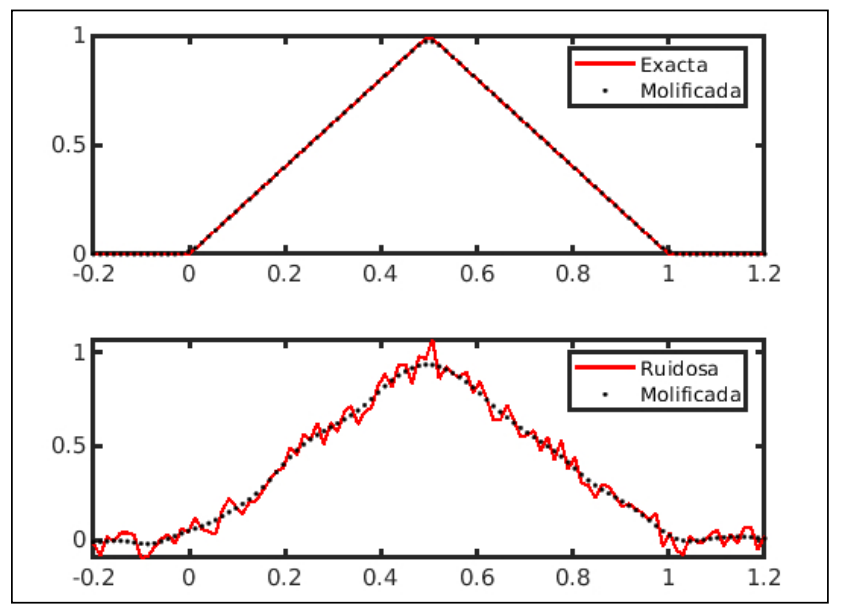

Figura 1. Molificación de función sin ruido y con ruido. en la implementación, pero, en todos los casos, se trata de métodos basados en convolución que son útiles para la regularización de problemas mal condicionados.

Hay pocas referencias de molificación bidimensional. Entre las mencionadas arriba, solamente en el estudio de Li \& Fu (2011) se aborda un problema con dominio en una franja de $\mathbb{R}^{2}$, pero se utiliza solamente la molificación unidimensional. El tema de la molificación en dos dimensiones se trata en la siguiente sección.

Molificación discreta bidimensional. El propósito de esta sección es presentar una generalización a dos dimensiones del operador de molificación unidimensional introducido en la sección anterior. El operador bidimensional de molificación discreta que definiremos enseguida fue introducido por Acosta \& Bürger (2012). Sin embargo, debe advertirse que la molificación bidimensional se está realizando por lo menos desde los años 90 (Zhan \& Murio, 1999; Zhan, et al., 2001).

Nuestro dominio es una malla uniforme bidimensional del plano $\mathbb{R}^{2}$ dada por $\left(x_{i}, y_{j}\right)=\left(x_{0}+i h, y_{0}+j k\right)$, donde $h$ y $k$ son los reales positivos que determinan tamaños de paso en cada dirección. Para una función $G$, definida en esta malla como $G\left(x_{i}, y_{j}\right)=G_{i j}$, se establece su molificación discreta como $\left(U_{\eta}^{2} G\right)_{i j}=\sum_{k=-\eta}^{\eta} \sum_{l=-\eta}^{\eta} \omega_{k} \omega_{l} G_{i+l, j+k}$, donde $\eta$ determina el soporte discreto y los pesos $\omega_{j}$ son los de la molificación unidimensional. Nótese que

$$
\left(J_{\eta}^{2} G\right)_{i j}=\sum_{k=-\eta}^{\eta} \omega_{k} \sum_{l=-\eta}^{\eta} \omega_{l} G_{i+l, j+k}=\sum_{k=-\eta}^{\eta} \omega_{k}\left(J_{\eta}^{x}\right)_{i, j+k}=J_{\eta}^{y}\left(J_{\eta}^{x} G\right)_{i j},
$$

donde los dos operadores $J_{\eta}^{x}$ y $J_{\eta}^{y}$ corresponden a la molificación unidimensional ya definida.

Las propiedades de aproximación de este operador aparecen en Acosta \& Bürger (2012) y en Echeverry (2018). Para las propiedades del operador de molificación bidimensional definido en la década de los 90 por el grupo liderado por Murio, recomendamos consultar Zhan \& Murio (1999).

Otro aspecto del método de molificación que ha suscitado mucho interés recientemente es el de las derivadas fraccionarias, las cuales se definen en la siguiente sección.

Derivadas fraccionarias. El estudio de las derivadas fraccionarias se denomina cálculo fraccional y sus orígenes se remontan a fines del siglo XVII. Importantes matemáticos de distintas épocas, como Leibniz, L'Hôpital, Johann Bernoulli, Euler, Abel, Riemann y Liouville, han contribuido al establecimiento de esta teoría. La calificación de estas derivadas como "ilustres" se justifica por la nómina de matemáticos destacados que ha ayudado a su nacimiento y evolución. Asimismo, nos atrevemos a afirmar que también son ilustres por el lugar destacado que ocupan en la matemática aplicada y porque estamos seguros de que su protagonismo aumentará en los próximos años. En el campo del cálculo fraccional sugerimos consultar a Oldham \& Spanier (2006), Miller \& Ross (1993), Podlubny (1999), Diethelm (2010) y Guo, et al. (2015). 
Recientemente, la investigación en cálculo fraccional se ha intensificado, pues hay evidencia de que varios modelos matemáticos mejoran cuando se cambian las derivadas usuales por las fraccionarias.

En el caso específico de difusión en un medio poroso y con fracturas, Fomin, et al. (2010) han establecido mediante experimentos que la difusión en el medio es anómala y que en lugar de las tradicionales leyes de Fick, es conveniente recurrir a ecuaciones de difusión con derivadas fraccionarias.

En el campo del modelamiento matemático de materiales viscoelásticos, se está encontrando que es preferible un modelo fraccional que uno con derivadas enteras (Diethelm, 2010).) La regla general es que si el proceso bajo estudio tiene propiedades asociadas con la memoria o la herencia, es útil intentar un modelo fraccional en lugar de uno tradicional.

A continuación definimos dos de las derivadas fraccionarias más conocidas. El orden de derivación en ambos casos es un número real positivo $\alpha$.

Derivada fraccionaria de Caputo. Para una función real $f$ suficientemente suave, la derivada de Caputo de orden $\alpha$ es

$$
{ }_{a}^{C} D_{t}^{\alpha} f(t)=\frac{1}{\Gamma(m-\alpha)} \int_{a}^{t} \frac{f^{(m)}(\tau)}{(t-\tau)^{\alpha-m+1}} d \tau,
$$

donde el entero $m$ cumple $m-1<\alpha \leq m$ y $\Gamma(z)=\int_{0}^{\infty} x^{z-1}$ $\exp (-x) d x$ es la función gamma, que es una generalización del factorial, pues si $n$ es un entero positivo, $\Gamma(n)=(n-1)$ !

Derivada de Riemann-Liouville. Sea $f$ una función real continua a trozos en $(0, \infty)$, integrable en cualquier subintervalo finito de $(0, \infty)$. Entonces para $t>0$, la integral fraccionaria de Riemann-Liouville de orden $\alpha$ de $f$ es

$$
{ }_{a}^{R L} D_{t}^{-\alpha} f(t)=\frac{1}{\Gamma(\alpha)} \int_{a}^{t}(t-\tau)^{\alpha-1} f(\tau) d \tau
$$

Para definir la derivada fraccionaria de RiemannLiouville de orden $\alpha$, utilizamos el entero $m$ tal que $m-1$ $\leq \alpha<m$ y tomamos $\lambda=m-\alpha$. La derivada fraccionaria de Riemann-Liouville de orden $\alpha$ de $f$ es

${ }_{a}^{R L} D_{t}^{\alpha} f(t)=\frac{d^{m}}{d t^{m}}\left({ }_{\alpha}^{R L} D_{t}^{-\lambda} f(t)\right)=\frac{1}{\Gamma(\lambda)} \frac{d^{m}}{d t^{m}} \int_{a}^{t}(t-\tau)^{\lambda-1} f(\tau) d \tau$.

El siguiente lema advierte sobre cuán distintas pueden ser las derivadas fraccionarias.

Lema 1. Para funciones constantes, la derivada fraccionaria de Caputo es cero y en cambio la derivada fraccionaria de Riemann-Liouville no es cero.

Dem: tomemos $0<\alpha<1, m=1$ y $f(t)=1$ para todo $t$. Debido a la presencia de la derivada en el integrando, la derivada fraccional de Caputo de orden $\alpha$ de $f$ es 0 . En cambio, la derivada de Riemann-Liouville de orden $\alpha$ de $f$ es la función

$$
g(t)=\frac{t^{-\alpha}}{\Gamma(1-\alpha)}
$$

Una anotación pertinente en este momento es: las derivadas de orden entero dependen del comportamiento "local" de la función y las derivadas fraccionarias se calculan con base en los valores de la función a todo lo largo del dominio de definición. Eso hace que a los operadores del cálculo fraccionario se les denomine operadores "no locales" o "con memoria".

\section{Problema inverso de advección-dispersión con derivada temporal fraccionaria}

El objetivo es estudiar la aplicación de la molificación como método de regularización para la solución del siguiente problema inverso: reconstrucción de la concentración de soluto en la frontera del dominio físico que es unidimensional semiinfinito. La derivada fraccionaria se toma en el sentido de Caputo y los coeficientes de advección y de dispersión son constantes. La información adicional que se requiere para enunciar correctamente el problema inverso y poderlo resolver consiste en una distribución de datos perturbados correspondientes a la concentración y al flujo en un punto interior del dominio para todo tiempo.

La configuración es unidimensional, lo cual significa que estamos ante una primera aproximación, tal como lo explican Benson, et al. (2000), de quienes citamos unas líneas que son pertinentes: "Una pregunta típica que se hace un hidrogeólogo que estudia el transporte de un contaminante es: ¿qué tan lejos y qué tan rápido avanzará el contaminante? Como primera aproximación, la mayoría de problemas de esta naturaleza se reducen a una ecuación unidimensional de advección-dispersión".

Advertimos que nos proponemos estudiar la solución numérica del problema inverso por molificación discreta $\mathrm{y}$ no consideramos el problema matemático de existencia y unicidad de soluciones.

Problemas directo e inverso. Enunciamos un problema inverso a partir del siguiente problema directo:

$$
\begin{array}{ccc}
{ }_{0}^{C} D_{t}^{\alpha} u+a u_{x} & =d u_{x x}, & 0<x, 0<t, \\
u(0, t) & =\rho(t), & 0 \leq t, \\
u(x, o) & =0, & 0<x,
\end{array}
$$

y se trabaja con la hipótesis adicional $u(x, t)$ acotada para $x$ $\rightarrow \infty$. La variable $u$ representa la concentración del soluto, $u_{x}$ es el flujo de dispersión, y las constantes positivas $a \mathrm{y} d$ representan la velocidad promedio del fluido y el coeficiente de dispersión, respectivamente. De ahora en adelante, a la derivada de Caputo ${ }_{0}^{C} D_{t}^{\alpha} u$ la denotamos como $D_{t}^{\alpha} u$.

El problema inverso de interés es:

$$
\begin{array}{ccc}
D_{t}^{\alpha} u(x, t)+a u_{x}(x, t) & =d u_{x x}(x, t), & 0<x, 0<t, \\
u(1, t) & =\rho(t), & 0<t, \quad \text { dato, } \\
u_{x}(1, t) & =\sigma(t), & 0<t, \quad \text { dato, } \\
u(0, t) & =\xi(t), & 0 \leq t, \text { incógnita, } \\
u_{x}(0, t) & =\beta(t), & 0 \leq t, \text { incógnita, } \\
u(x, 0) & =0, & 0<x .
\end{array}
$$

La información adicional consiste en las medidas interiores en $x=1$ para la concentración y el flujo de dispersión. Para hacer realista la situación, suponemos que la información adicional no se conoce exactamente y sus aproximaciones $\rho^{\varepsilon}$ y $\sigma^{\varepsilon}$ satisfacen los estimados $\left\|\rho-\rho^{\varepsilon}\right\|_{\infty}$ $<\varepsilon$ y $\left\|\sigma-\sigma^{\varepsilon}\right\|_{\infty}<\varepsilon$, donde $\varepsilon$ representa el máximo nivel de perturbaciones en los datos.

Se trata de un problema mal condicionado según el siguiente teorema. 
Teorema 1. Aproximar $\xi y \beta$ a partir de la ecuación diferencial $y$ las mediciones interiores adicionales $\rho^{\varepsilon} y$ $\sigma^{\delta}$ es un problema gravemente mal condicionado en las componentes de alta frecuencia.

Dem: proponemos solamente un esbozo. Suponemos que todas las funciones dependientes del tiempo con las que trabajamos se pueden extender a toda la recta real. Aplicamos la transformada de Fourier con respecto a $t \mathrm{y}$ llegamos al sistema

$$
\left[\begin{array}{l}
\widehat{\xi(\omega)} \\
\hat{\beta(\omega)}
\end{array}\right]=\left[\begin{array}{cc}
\cosh \mu \nu & \frac{1}{\mu \nu} \sinh \mu \nu \\
\mu \nu \sinh \mu \nu & \cosh \mu \nu
\end{array}\right]\left[\begin{array}{c}
\widehat{\rho(\omega)} \\
\widehat{\sigma(\omega)}
\end{array}\right] .
$$

La variable en el espacio de frecuencias es $\omega, \mu=\mid a^{2}$ $+\left.4 d(i \omega)^{\alpha}\right|^{1 / 2}$ y $v=\cos (\theta / 2)+i \sin (\zeta \theta / 2)$, donde $\theta=\arg$ $\left(a^{2}+4 d(i \omega)^{\alpha}\right)=\arctan \left(\frac{4 d|\omega| \omega^{\alpha} \sin \left(\frac{3 \pi \alpha}{2}\right)}{a^{2}+4 d \mid \omega \omega^{\alpha} \cos \left(\frac{3 \pi \alpha}{2}\right)}\right)$. Además, $\theta=\arg \left(a^{2}+\right.$ $\left.4 d(i \omega)^{\alpha}\right)=\tan ^{-1}\left(\frac{4 d|\omega| \alpha_{\sin }\left(\frac{3 \pi \alpha}{2}\right)}{a^{2}+\left.4 d|\omega|\right|^{\alpha} \cos \left(\frac{\zeta \pi \alpha}{2}\right)}\right)$. Además, $(i \omega)^{\alpha}=|\omega|^{\alpha} \exp \left(\frac{i \zeta \pi \alpha}{2}\right)$ $\operatorname{con} \zeta=\operatorname{sgn}(\omega)$. Se cumple que si $|\omega| \rightarrow \infty$, entonces

$$
|\cosh \mu \nu|=\left|\cosh \left(\left|a^{2}+4 d(i \omega)^{\alpha}\right|^{\frac{1}{2}}\left(\cos \frac{\theta}{2}+i \sin \frac{\zeta \theta}{2}\right)\right)\right| \rightarrow \infty
$$

$\mathrm{y}$

$$
|\sinh \mu \nu|=\left|\sinh \left(\left|a^{2}+4 d(i \omega)^{\alpha}\right|^{\frac{1}{2}}\left(\cos \frac{\theta}{2}+i \sin \frac{\zeta \theta}{2}\right)\right)\right| \rightarrow \infty,
$$

de donde se concluye el mal condicionamiento anunciado (Mejía \& Piedrahita, 2017).

La molificación y la derivada fraccionaria. La presencia de la derivada dentro de la integral en la derivada fraccionaria de Caputo anuncia la posibilidad de un problema mal condicionado cuando los datos no se conocen exactamente. Seguimos a Murio (2008) para el esquema implícito de discretización de la derivada fraccionaria y a Murio \& Mejía (2008b) para la combinación de la derivada fraccionaria con la molificación.

Lema 2. Sea g una función continuamente diferenciable. Consideremos la cuadratura con parámetro de discretización temporal $k$ de la derivada de Caputo de g dada por la fórmula

$$
C^{\alpha} g(t)=\sigma_{\alpha, k} \sum_{i=0}^{\left\lfloor\frac{t}{k}\right\rfloor} \omega_{i}^{(\alpha)}(g(t-i k+1)-g(t-i k)),
$$

donde [.] denota la función parte entera o piso, $\sigma_{\alpha, k}=\frac{1}{\Gamma(1-\alpha)} \frac{1}{1-\alpha} \frac{1}{k^{\alpha}}$ $y \omega_{i}^{(\alpha)}=i^{1-\alpha}-(i-1)^{1-\alpha}$.

Entonces esta cuadratura satisface la aproximación de primer orden

Dem: Ver Murio (2008.)

$$
D_{t}^{\alpha} g(t)=C^{\alpha} g(t)+O(k) .
$$

El problema estabilizado por molificación se define para las variables $v=J_{\delta} u$ y $v_{x}=J_{\delta} u_{x}$ y es el siguiente:

$$
\begin{array}{ccc}
D_{t}^{\alpha} v(x, t)+a v_{x}(x, t) & =d v_{x x}(x, t), & 0<x, 0<t, \\
v(1, t) & =J_{\delta} \rho^{\varepsilon}(t), & 0<t, \quad \text { dato, } \\
v_{x}(1, t) & =J_{\delta} \sigma^{\varepsilon}(t), & 0<t, \quad \text { dato, } \\
v(0, t) & =J_{\delta} \xi(t), & 0 \leq t, \text { incógnita, } \\
v_{x}(0, t) & =J_{\delta} \beta(t), & 0 \leq t, \text { incógnita, } \\
v(x, 0) & =0, & 0 \leq x,
\end{array}
$$

donde $D_{t}^{\alpha} v=D_{t}^{\alpha} J_{\delta} u=J_{\delta} D_{t}^{\alpha} u$.

Enseguida se enuncia un esquema de diferencias finitas con sentido de marcha en la dirección del espacio. Esta es una técnica que ha probado su efectividad en la solución de problemas inversos (Murio, 2002; Mejía, et al., 2011; Mejía \& Murio, 2008a; Mejía \& Murio, 2008b; Garshasbi \& Dastour, 2015).

Los parámetros de discretización son: números reales positivos $k, h$ y números enteros positivos $N, M$ tales que $N k=\tau$ es el tiempo final y $M h=1$. La malla espacial es $x=(m h)_{m=0, \ldots, M}$ y el vector de instantes de tiempo es $t=(n k)_{n=0, \ldots, N}$. Denotamos $R_{m}{ }^{n}, W_{m}{ }^{n y}$ y $Q_{m}{ }^{n}$ a las aproximaciones calculadas para la concentración de soluto $v(m h, n k)$, el flujo molificado $v_{x}(m h, n k)$ y la derivada temporal fraccionaria de la concentración de soluto $D_{t}^{a} v(m h, n k)$ respectivamente. El esquema con sentido de marcha en la dirección espacial es el siguiente:

$$
\begin{gathered}
R_{m-1}^{n}=R_{m}^{n}-h W_{m}^{n}, \\
W_{m-1}^{n}=\left(1-\frac{a}{d} h\right) W_{m}^{n}-\frac{h}{d} Q_{m}^{n},
\end{gathered}
$$

donde $m=M, M-1, \ldots, 1$ y $n=1, \ldots, N$. Las aproximaciones buscadas son $R_{0}^{n}$ y $W_{0}^{n}$ para $n=1, \ldots, N$.

Para poder calcular con este esquema es indispensable un método numérico de solución del problema directo en $[0,1]$ pues esa es la forma de obtener los datos de inicio. De esto se ocupa la próxima sección.

Generación de los datos para la solución numérica del problema directo. Lo usual es que no se disponga de solución exacta para el problema directo. De hecho, en las industrias y talleres es muy frecuente que se enuncien problemas inversos y se resuelvan en el computador debido a la imposibilidad de medir en una de las fronteras, o en el interior del equipo con el que se está trabajando, por ejemplo, una caldera o una turbina.

El problema directo de advección difusión con derivada temporal fraccionaria asociado al problema molificado es

$$
\begin{array}{ccc}
D_{t}^{\alpha} v(x, t)+a v_{x}(x, t) & =d v_{x x}(x, t), & 0<x<1,0<t<\tau, \\
v(0, t) & =v_{l}(t), & 0<t \leq \tau, \\
v(1, t) & =v_{r}(t), & 0<t \leq \tau, \\
v(x, 0) & =f(x), & 0 \leq x \leq 1 .
\end{array}
$$

Para este problema directo se enuncia un método implícito de solución por diferencias finitas que tiene en cuenta el método de aproximación de la derivada fraccionaria del Lema 2. Los detalles se pueden consultar en Mejía \& Piedrahita (2017.)

\section{Teorema 2 (Convergencia)}

La aproximación de la concentración obtenida con el esquema de marcha en la dirección espacial para la frontera activa $x=0$, converge a la concentración exacta cuando el paso temporal y el tamaño de las subdivisiones espaciales tienden a cero.

Experimento numérico. El experimento consiste en la recuperación de una concentración con forma de paso unitario. Más precisamente, la concentración en la frontera izquierda (por identificar) es la función

$$
w_{0}(t)= \begin{cases}1, & 0.2 \leq t \leq 0.6, \\ 0, & t \notin[0.2,0.6],\end{cases}
$$


y la concentración en la frontera derecha es $w_{1}(t)=0$. Los errores absolutos de la Tabla 2 se obtuvieron con la norma RMS (root mean square), que se define así: $x=\left(x_{1}, x_{2}\right.$, $\left.\ldots, x_{n}\right)$ si entonces $R M S(x)$ es la raíz cuadrada de la media aritmética de los cuadrados de los $x_{j}$. La Figura 2 ilustra la calidad de la identificación. Se obtuvo con los parámetros de discretización $h=\frac{1}{100} \mathrm{y} k=\frac{1}{128}$.

En la Tabla 2 se presenta un informe de resultados numéricos para dos valores de $\alpha$. Sirve para observar la dependencia del error de los parámetros de discretización $h$ $\mathrm{y} k \mathrm{y}$, por lo tanto, para verificar el teorema de convergencia. Nótese que la concentración en el borde $x=0$ es una función de $t$ y que los errores son menores para los menores valores del parámetro de discretización temporal $k$.

\section{Problema inverso de identificación de término fuente}

En esta sección presentamos los resultados obtenidos para un problema inverso de identificación de término fuente en una ecuación de difusión en la que la derivada temporal es fraccionaria en el sentido de Caputo y la configuración espacial es bidimensional.

El problema directo. El problema directo de difusión de interés para nosotros corresponde al seguimiento de la concentración de un contaminante en un acuífero en el que la dispersión o difusión es dominante y, por lo tanto, no se tiene en cuenta la advección. El término fuente de la ecuación cumple la regla de variables separables en la que el factor dependiente de espacio es la magnitud o intensidad de la descarga del contaminante y el factor dependiente del tiempo es un coeficiente de atenuación. El término de dispersión o difusivo es un operador diferencial denotado con la letra $L$ y se explica más adelante. El problema directo de valor inicial y valores en la frontera son

$$
\begin{array}{cccc}
D_{t}^{\alpha} u(z, t)-(L u)(z, t)= & p(t) f(z), & z \in \Omega & t \in(0, T) \\
u(z, t) & =0, & z \in \partial \Omega & t \in(0, T) \\
u(z, 0) & =0, & z \in \Omega, &
\end{array}
$$

donde $\Omega \subset \mathbb{R}^{d}, \bar{\Omega}$ es su clausura, la derivada temporal fraccionaria es en el sentido de Caputo, la difusión es

$$
L u(z)=\sum_{i=1}^{d} \frac{\partial}{\partial z_{i}}\left(\sum_{j=1}^{d} a_{i j}(z) \frac{\partial}{\partial z_{j}} u(z)\right)+c(z) u(z), z \in \Omega
$$

y los coeficientes en $L$ satisfacen las siguientes relaciones: $a_{i j}=a_{j i} \in C^{1}(\bar{\Omega}), \sum_{i, j=1}^{d} a_{i j} \zeta_{i} \zeta_{j}>\theta \sum_{i=1}^{d}\left|\zeta_{i}\right|^{2}, \theta>0, c(z) \leq 0, c \in C(\bar{\Omega})$.

Con estas condiciones el operador $-L$ es simétrico y uniformemente elíptico, definido en $D(-L)=H^{2}(\Omega) \cap H_{0}^{1}(\Omega)$.

El problema inverso. Nos interesa identificar el factor $\mathrm{f}(\mathrm{z})$ en el término fuente, lo cual exige que tengamos alguna información adicional. En nuestro caso se trata de la distribución espacial de la concentración $u(z, T)=q(z)$ en el interior del dominio para un tiempo futuro T. Más aun, suponemos que la información adicional es una medición y está sujeta a errores. Por lo tanto, en lugar de la distribución exacta $q$ disponemos de una aproximación $q^{\varepsilon}$ que cumple con $\left\|q-q^{\varepsilon}\right\|_{\infty} \leq \varepsilon$.

Existencia de la solución. Obtenemos de Ma, et al. (2018) y Sakamoto \& Yamamoto (2011) una expansión
Tabla 2. Reconstrucción de $u(0, t)$ con ruido máximo $\varepsilon=0,05$

\begin{tabular}{cccc}
\hline $\boldsymbol{h}$ & $\mathbf{k}$ & $\begin{array}{c}\text { Error absoluto, } \\
\boldsymbol{\alpha}=\mathbf{0 , 5}\end{array}$ & $\begin{array}{c}\text { Error absoluto, } \\
\boldsymbol{\alpha}=\mathbf{0 , 9}\end{array}$ \\
\hline $1 / 50$ & $1 / 128$ & 0,0875 & 0,1256 \\
$1 / 50$ & $1 / 256$ & 0,0754 & 0,1051 \\
$1 / 100$ & $1 / 128$ & 0,0958 & 0,1216 \\
$1 / 100$ & $1 / 256$ & 0,0830 & 0,1054 \\
$1 / 100$ & $1 / 512$ & 0,0809 & 0,0982 \\
\hline
\end{tabular}

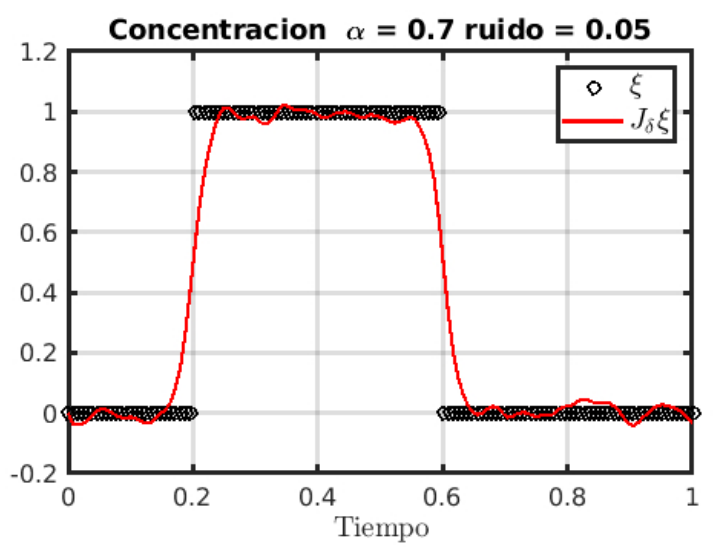

Figura 2. Exacta: línea con diamantes; calculada: línea continua

en serie para la solución del problema directo. Está basada en las funciones propias del operador $-L$ y en la función de Mittag-Leffler de dos parámetros positivos $\alpha$ y $\beta$ dada por

$$
E_{\alpha, \beta}(z)=\sum_{k=0}^{\infty} \frac{z^{k}}{\Gamma(\alpha k+\beta)} .
$$

Nótese que esta función es una generalización de la función exponencial. Denotemos por $\left\{\chi_{n}\right\}_{n \in \mathbb{N}}$ las funciones propias de $-L$ y $\left\{\lambda_{n}\right\}_{n \in \mathbb{N}}$ a sus valores propios. Además, denotamos (..,) al producto interno en $L^{2}(\Omega)$. La solución del problema directo es

$u(z, t)=\sum_{n=1}^{\infty} f_{n}\left[\int_{0}^{t} p(\tau)(t-\tau)^{\alpha-1} E_{\alpha, \alpha}\left(-\lambda_{n}(t-\tau)^{\alpha}\right) d \tau\right] \chi_{n}(z)$, donde $f_{n}=\left(f, \chi_{n}\right)$. Por lo tanto,

$u(z, T)=\sum_{n=1}^{\infty} f_{n}\left[\int_{0}^{T} p(\tau)(T-\tau)^{\alpha-1} E_{\alpha, \alpha}\left(-\lambda_{n}(T-\tau)^{\alpha}\right) d \tau\right] \chi_{n}(z)=q(z)$.

Denotemos $Q_{n}(t)=\int_{0}^{t} p(\tau)(t-\tau)^{\alpha-1} E_{\alpha, \alpha}\left(-\lambda_{n}(t-\tau)^{\alpha}\right) d \tau$. Entonces, $q(z)=\sum_{n=1}^{\infty} f_{n} Q_{n}(T) \chi_{n}(z)$, es decir, sus coeficientes de Fourier son $q_{n}=f_{n} Q_{n}(T)$. Para $\gamma \in \mathbb{R}$ definimos el espacio

$$
D\left((-L)^{\gamma}\right)=\left\{\psi \in L^{2}(\Omega): \sum_{n=1}^{\infty} \lambda_{n}^{2 \gamma}\left|\left(\psi, \chi_{n}\right)\right|^{2}<\infty\right\} .
$$

Es un espacio de Hilbert con la norma $\|\psi\|_{D\left((-L)^{r}\right)}=$ $\left(\sum_{n=1}^{\infty} \lambda_{n}^{2 \gamma}\left|\left(\psi, \chi_{n}\right)\right|^{2}\right)^{\frac{1}{2}}$.

Para la solución del problema inverso definimos el operador

$(K f)(z)=\sum_{n=1}^{\infty} f_{n}\left[\int_{0}^{t} p(\tau)(t-\tau)^{\alpha-1} E_{\alpha, \alpha}\left(-\lambda_{n}(t-\tau)^{\alpha}\right) d \tau\right] \chi_{n}(z)$ y buscamos una función $f_{\eta}^{\varepsilon}$ que minimiza al funcional $H f=$ $\left\|K f-J_{\eta}^{2} q^{\varepsilon}\right\|_{2}^{2}$. 


\section{Teorema 3}

Supongamos que $p \in C([0, T])$ y satisface $p(t) \geq p_{0}>0$ para todo $t \in[0, T]$. Supongamos además $\left\|q-q^{\varepsilon}\right\|_{\infty}<\varepsilon$ y existen $m \in \mathbb{R}^{+}$y $E \in \mathbb{R}$ tales que $\|f\|_{D\left((-L)^{2}\right)} \leq E$. Entonces

$$
\left\|f_{\eta}^{\varepsilon}-f\right\|_{2} \leq C_{1}(\delta+\varepsilon)^{\frac{m}{m+2}}
$$

donde $C_{1}$ es una constante que puede depender de $\alpha, T, \lambda_{1} \mathrm{y}$ la medida del dominio $\mu(\Omega)$. Además, $\delta$ es el parámetro de molificación asociado con $\eta$.

Dem: la solución está dada por

$$
\left.\boldsymbol{f}_{\eta}^{\varepsilon}(\mathbf{z})=\sum_{n=1}^{\infty} \frac{\left(U_{\eta}^{2} q^{\varepsilon}\right)_{n}}{Q_{n}(T)} \chi_{n}(\mathbf{z}) \text {, donde } U_{\eta}^{2} q^{\varepsilon}\right)_{n}=\left(U_{\eta}^{2} q^{\varepsilon}, \chi_{n}\right)
$$

(Echeverry \& Mejía, 2018).

El método numérico. La solución del problema inverso se realiza con diferencias finitas y requiere de la solución del problema directo. El método implícito utilizado está descrito en Mejía \& Piedrahita (2018), pero no tiene todavía un sistema eficiente de condiciones de borde para la molificación bidimensional, aspecto en el que estamos trabajando todavía. Por tal razón, la medida de errores en el experimento que presentamos enseguida, se realiza sobre puntos interiores de la malla. Los puntos fronterizos y los inmediatamente siguientes se dejan por fuera en el cálculo del error. Utilizamos la norma RMS.

El experimento numérico en el que se basan las figuras siguientes consiste en la ecuación diferencial fraccional

$$
D_{t}^{\alpha} u(z, t)-(L u)(z, t)=p(t) f(z)
$$

donde $z=(x, y) \in \Omega, \mathrm{t} \in(0, T)$ y $L u(z, t)$ está dado por

$$
\boldsymbol{L u}(x, y, t)=\frac{\partial}{\partial x}\left(a_{11}(x, y) \frac{\partial}{\partial x} \boldsymbol{u}(x, y, t)\right)+\frac{\partial}{\partial y}\left(a_{22}(x, y) \frac{\partial}{\partial y} \boldsymbol{u}(x, y, t)\right),
$$

con $a_{11}(x, y)=x^{2}+1, a_{22}(x, y)=y^{2}+1, p(t)=1+\exp (-(t$ $+\alpha)$ ), y el ingrediente por identificar es $f(x, y)=\sin 3 \pi x \sin$ $3 \pi y$. El dominio $\Omega$ es un rectángulo unitario y $T=1$. Como parámetros de discretización usamos $\mathrm{h}=1 / 32$ tanto en $x$ como en $y$ y $\mathrm{k}=1 / 10$ en tiempo. El máximo nivel de ruido en los datos es $\varepsilon=0,05$.

En primer lugar, en la Figura 3 se ilustra la importancia de incluir la molificación para lograr un método estable. En segundo lugar, en la Figura 4 se ilustra la calidad de la identificación que se logra con una adecuada molificación.

Por último, en la Tabla 3 se presentan los resultados de otros experimentos que sirven para verificar la convergencia del algoritmo. Los errores en la identificación de $f$ se calculan con la norma RMS.

\section{Discusión y conclusiones}

En este artículo verificamos la utilidad de los operadores de molificación en aplicaciones a problemas inversos en una y dos dimensiones basados en ecuaciones difusivas en las que la derivada temporal es fraccionaria. También expusimos argumentos para justificar el apelativo de "ilustres" para las derivadas fraccionarias. Creemos haber establecido que las derivadas fraccionarias serán cada vez más importantes en la matemática aplicada y que la molificación discreta es un método de regularización adecuado para resolver problemas inversos en los que esas derivadas participan.
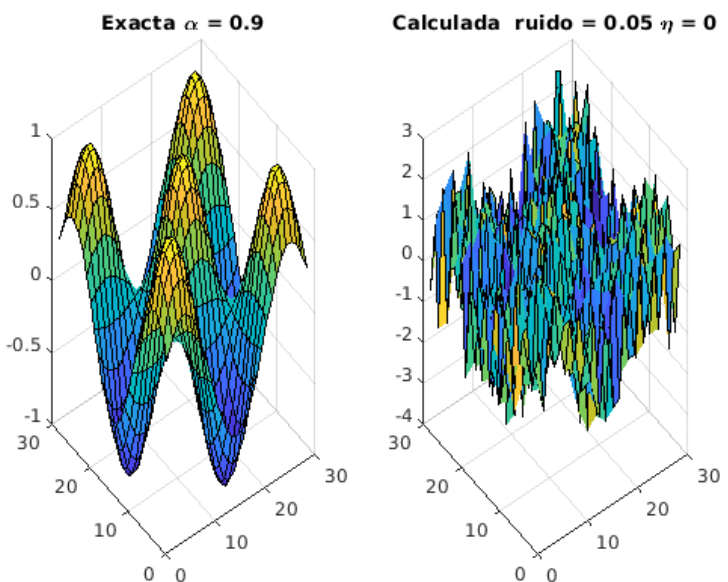

Figura 3. A. Identificación fallida del factor $\mathrm{f}$ por falta de molificación
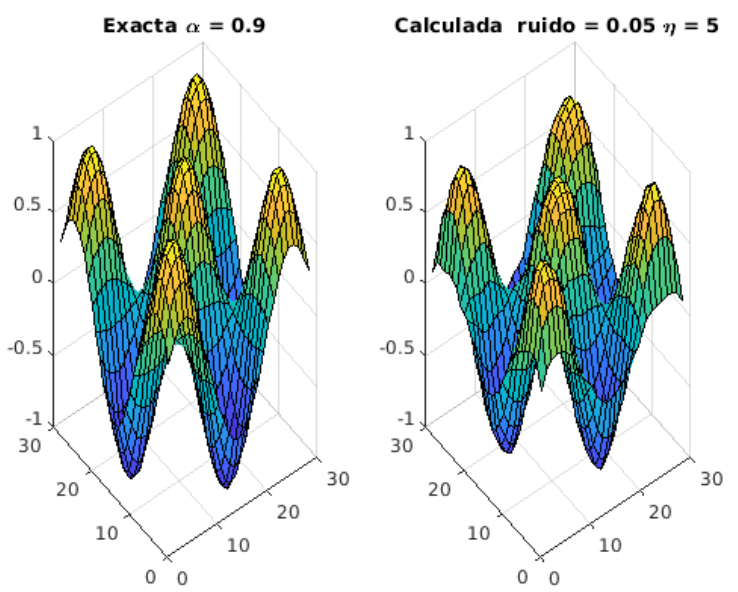

Figura 4. Identificación de factor $\mathrm{f}$ con $\mathrm{h}=1 / 32$ y $\mathrm{k}=1 / 10$

Tabla 3. Identificación del factor $f$ para $\varepsilon=0,05$

\begin{tabular}{ccccc}
\hline $\boldsymbol{h}$ & \multicolumn{4}{c}{$\boldsymbol{\alpha}$} \\
\hline En x y en y & 0,1 & 0,5 & 0,7 & 0,9 \\
$1 / 30$ & 0,1157 & 0,1186 & 0,1203 & 0,1190 \\
$1 / 40$ & 0,0824 & 0,0874 & 0,0888 & 0,0883 \\
$1 / 50$ & 0,0734 & 0,0742 & 0,0746 & 0,0754 \\
$1 / 60$ & 0,0657 & 0,0735 & 0,0741 & 0,0712 \\
\hline
\end{tabular}

Seguiremos intentando ayudar en el desarrollo de este campo de investigación de análisis numérico, con especial énfasis en el estudio de ecuaciones diferenciales fraccionarias en las que las derivadas fraccionarias están en las variables espaciales. Asimismo, aspiramos a mejorar el operador de molificación bidimensional en lo que respecta a las condiciones de borde. Por último, nos proponemos enfrentar problemas inversos basados en ecuaciones 
difusivas no lineales en las que hay derivadas fraccionarias. Recientemente, consideramos ecuaciones de adveccióndispersión con término fuente no lineal y derivada temporal fraccionaria (Mejía \& Piedrahita, 2019), y el plan es continuar explorando este tema y enfrentar nuevos problemas en el futuro inmediato.

\section{Agradecimientos}

El autor agradece el apoyo de la Universidad Nacional de Colombia a través del proyecto "Operadores no locales y con memoria”, código Hermes 33154.

\section{Referencias}

Acosta, C. D. \& Bürger, R. (2012). Difference schemes stabilized by discrete mollification for degenerate parabolic equations in two space dimensions, IMA J. Numer. Anal. 32: 1509-1540.

Acosta, C. D., Bürger, R., Mejía, C. E. (2012). Monotone difference schemes stabilized by discrete mollification for strongly degenerate parabolic equations, Numer. Meth. Partial Diff. Eqns. 28: 38-62.

Acosta, C. D., Bürger, R., Mejía, C. E. (2014). A stability and sensitivity analysis of parametric functions in a sedimentation model, DYNA Vol. 81 (183): 22-30.

Acosta, C. D. Bürger, R., Mejía, C. E. (2015). Efficient parameter estimation in a macroscopic traffic flow model by discrete mollification, Transportmetrica A: Transport Science. 11 (8): 702-715.

Acosta, C. D. \& Mejía, C. E. (2014). Stable computations by discrete mollification, Bogotá, Universidad Nacional de Colombia. p. 108.

Acosta, C. D. \& Mejía, C. E. (2008). Stabilization of explicit methods for convection diffusion equations by discrete mollification. Computers Math. Applic. 55: 368-380.

Benson, D. A., Wheatcraft, S. W., Meerschaert M. M. (2000). Application of a fractional advection-dispersion equation. Water Resources Research. 36: 1403-1412.

Diethelm, K. (2010). The analysis of fractional differential equations. An application-oriented exposition using differential operators of Caputo type. Berlin, Springer. p. 247.

Echeverry, M. D. (2018). Fractional differential equations and inverse problems. Tesis de Doctorado en preparación, Universidad Nacional de Colombia, sede Medellín.

Echeverry, M. D. \& Mejía, C. E. (2018). A two dimensional discrete mollification operator and the numerical solution of an inverse source problem. Axioms. 7 (4): 89. Doi: 0.3390/axioms 7040089

Fomin, S., Chugunov, V., Hashida, T. (2010). Application of fractional differential equations for modeling the anomalous diffusion of contaminant from fracture into porous rock matrix with bordering alteration zone. Transp. Porous Med. 81: 187-205.

Friedrichs, K. O. (1944). The identity of weak and strong extensions of differential operators. Trans. AMS. 55: 132-151.

Garshasbi, M. \& Dastour, H. (2015). Estimation of unknown boundary functions in an inverse heat conduction problem using a mollified marching scheme. Numer. Algor. 68: 769-790.
Guo, B., Pu, X., Huang, F. (2015). Fractional partial differential equations and their numerical solutions. New Jersey, World Scientific. p. 336.

Hao, D. N. (1994). A mollification method for ill-posed problems. Numer. Math. 68: 469-506.

Li, Z. \& Fu, C. (2011). A mollification method for a Cauchy problem for the Laplace equation. Applied Mathematics and Computation. 217: 9209-9218.

Ma, Y.-K., Prakash, P., Deiveegan, A. (2018). Generalized Tikhonov methods for an inverse source problem of the time-fractional diffusion equation. Chaos, Solitons and Fractals. 108: 39-48.

Manselli, P. \& Miller K. (1980). Calculations of the surface temperature and heat flux on one side of a wall from measurements on the opposite side. Ann. Math. Pura Appl. 123: 161-183.

Mejía, C. E. (2007). Sobre el método de molificación. Trabajo presentado como requisito parcial para promoción a profesor titular, Medellín, Universidad Nacional de Colombia.

Mejía, C. E., Acosta, C. D., Saleme, K. (2011). Numerical identification of a nonlinear diffusion coefficient by discrete mollification. Computers Math. Applic. 62: 2187-2199.

Mejía, C. E. \& Murio, D. A. (1995). Numerical identification of diffusivity coefficient and initial condition by discrete mollification. Computers Math. Applic. 30: 35-50.

Mejía, C. E. \& Murio, D. A. (1996). Numerical solution of generalized inverse heat conduction problem by discrete mollification. Computers Math. Applic. 32: 33-50.

Mejía, C. E. \& Piedrahita, A. (2019). A numerical method for a time fractional advection-dispersion equation with a nonlinear source term. J. Appl. Math. Comput. Doi: 10.1007/s12190-019-01266-x

Mejía, C. E. \& Piedrahita, A. (2017). Solution of a time fractional inverse advection-dispersion problem by discrete mollification. Revista Colombiana de Matemáticas. 51 (1): 83-102.

Mejía, C. E. \& Piedrahita, A. (2018). A finite difference approximation of a two dimensional time fractional advectiondispersion problem. https://arxiv.org/abs/1807.07393

Miller, K. S. \& Ross, B. (1993). An introduction to the fractional calculus and fractional differential equations. New York, John Wiley \& Sons. p. 366.

Murio, D. A. (1993). The mollification method and the numerical solution of ill-posed problems. New York, Estados Unidos. John Wiley and Sons. p. 254.

Murio, D. A. (2002). Mollification and space marching. En K. Woodbury (ed.), Inverse Engineering Handbook. Boca Raton, Estados Unidos. CRC Press. p. 466.

Murio, D. A. (2007). Stable numerical solution of a fractionaldiffusion inverse heat conduction problem. Computers Math. Applic. 53: 1492-1501.

Murio, D. A. (2008). Implicit finite difference approximation for time fractional diffusion equations. Computers Math. Applic. 56: 1138-1145.

Murio, D. A. \& Mejía, C. E. (2008a). Generalized time fractional IHCP with Caputo Fractional Derivatives, Journal of Physics: Conference Series. 135: 012074. 
Murio, D. A. \& Mejía, C. E. (2008b). Source Terms Identification for Time Fractional Diffusion Equation. Revista Colombiana de Matemáticas. 42 (1): 25-46.

Oldham, K. B. \& Spanier, J. (2006). the fractional calculus. Theory and applications of differentiation and integration to arbitrary order. Mineola, New York, Estados Unidos. Dover Publications. p. 234.

Podlubny, I. (1999). Fractional differential equations. Academic Press. p. 340.

Sakamoto, K. \& Yamamoto, M. (2011). Initial value/boundary value problems for fractional diffusion-wave equations and applications to some inverse problems. J. Math. Anal. Appl. 382: 426-447.
Shi, C., Wang, C., Wei, T. (2016). Convolution regularization method for backward problems of linear parabolic equations. Applied Numerical Mathematics. 108: 143-156.

Zhan, S. \& Murio, D. A. (1999). Surface fitting and numerical gradient computations by discrete mollification. Computers Math. Applic. 37: 85-102.

Zhan, S., Coles, C., Murio, D. A. (2001). Automatic numerical solution of generalized 2-D IHCP by discrete mollification, Computers Math. Applic. 41: 15-38. 